\title{
Impact of Neostigmine and Sugammadex on Time to Leaving the Operating Room in a Community Hospital
}

Julie John, PharmD ${ }^{1}$; Greg Perry, PharmD, BCPS-AQID'; Jeremie Perry, MD ; Viktoria Guttenberg, PharmD Candidate'; Nicole Asonganyi, PharmD Candidate'; Sana Laheji, PharmD2; Jaffar Raza, PharmD, MBA'; Ronald G. Hall, PharmD, MSCS

${ }^{1}$ Hendrick Medical Center; ${ }^{2}$ Texas Tech University Health Sciences Center, School of Pharmacy

\begin{abstract}
Purpose: The purpose of this study was to evaluate the impact of sugammadex on operating room (OR) times versus neostigmine in patients recovering from rocuronium or vecuronium induced neuromuscular blockade.

Methods: This retrospective cohort study evaluated patients 18 years or older with an American Society of Anesthesiologists (ASA) physical status of I-III who received sugammadex or neostigmine (January-October 2017) for reversal of rocuronium or vecuronium at a 500 bed, community hospital. Patients who were pregnant or breastfeeding were excluded. The primary outcome measure was the time from sugammadex or neostigmine administration to OR exit. The primary outcome was evaluated using a linear regression model adjusting for inpatient procedures, age, sex, body mass index, and ASA score. Secondary outcomes included the incidence of bradycardia as well as nausea and vomiting.

Results: The baseline characteristics of the patients in the cohort (sugammadex=134, neostigmine=143) were similar. The median time from drug administration to OR exit was similar for neostigmine and sugammadex (16 vs. 15.5 minutes, $p=0.11$ ). Sugammadex had a statistically significant reduction in time from drug administration to OR exit (coefficient -2.7 minutes, $95 \%$ confidence interval -5.2 to -0.2 minutes) in the multivariable linear regression model. Sugammadex had lower rates of bradycardia (5.6 vs. $2.2 \%$ ) or nausea and vomiting (18 vs. 11\%) that did not reach statistical significance.

Conclusions: Sugammadex had statistically shorter OR exit times after drug administration in the cohort. The mean 2.7 minute benefit is unlikely to be clinically meaningful and limits its application in practice unless larger cohorts detect a benefit due to a significant reduction in adverse events.
\end{abstract}

Keywords: sugammadex, neostigmine, neuromuscular blocker reversal, anesthesia, surgery

\section{Introduction}

The United States (US) Food and Drug Administration approved sugammadex in 2015. This is significant as sugammadex is the first new agent in decades for neuromuscular blocker (NMB) reversal in the US. A meta-analysis reported that sugammadex produced quicker reversal of NMB (10.2 minutes) along with decreased risks of bradycardia ( $\mathrm{RR} 0.6,95 \% \mathrm{Cl} 0.07$ to 0.34 ), postoperative nausea and vomiting (RR $0.52,95 \% \mathrm{Cl} 0.28-0.97$ ), and postoperative residual paralysis ( $\mathrm{RR} 0.40,95 \% \mathrm{Cl} 0.28$ to $0.57)$ when compared to neostigmine. ${ }^{1}$ Another meta-analysis reported faster discharge times from the operating room (OR) (mean difference 22 minutes) and the post-anesthesia care unit (PACU) (mean difference 17 minutes) for sugammadex when compared with neostigmine. ${ }^{2}$ However, the application of these findings into routine clinical practice are limited by the drug acquisition costs of sugammadex and whether these reductions in OR times can be replicated in community hospital settings. Potential reasons for differences in sugammadex's effectiveness between community and the data from randomized, controlled trials in academic hospitals include the types of surgical cases, staffing levels, and/or monitoring protocols.

Corresponding author: Ronald Hall, PharmD, MSCS

Texas Tech University Health Sciences Center, School of Pharmacy, 5920 Forest Park Rd, Suite 424, Dallas, TX 75235, Phone: 214-358-9009; Fax: 214-654-9707

E-mail: Ronald.hall@ttuhsc.edu
The meta-analysis suggesting quicker OR and PACU discharge times with sugammadex compared with neostigmine included very few studies with limited numbers of patients. ${ }^{2}$ Specifically, the time to OR discharge analysis was only able to use two of the six included studies $(n=108)$ and the PACU discharge analysis used four studies $(n=353)$. This meta-analysis included only randomized controlled trials. While randomized controlled trials are the gold standard of evidence-based medicine, they may not reflect a drug's effectiveness in a community hospital setting. Randomized, controlled trials have rigorous protocols and are often conducted in large, academic medical centers that do not share many characteristics with community hospitals. This means that we do not know how much of sugammadex's beneficial adverse event profile and shorter OR times will translate into routine surgical practice. If these findings can be replicated in the community hospital setting, then sugammadex may be beneficial as it would decrease costs related to OR times and adverse events.

This study was conducted to determine if sugammadex would produce shorter OR times compared to neostigmine for NMB reversal in a community hospital that performs a wide variety of elective and non-elective procedures. We addressed this question by conducting a retrospective cohort study of sugammadex versus neostigmine in patients receiving NMB for a surgical procedure at a community hospital. We hypothesized the use of sugammadex would result in shorter times from 
administration of the $\mathrm{NMB}$ reversal agent to OR exit than neostigmine.

\section{Materials and Methods}

This retrospective cohort study included patients who underwent a surgical procedure at a 500-bed community hospital from January 1, 2017 to October 31, 2017. Anesthesia was induced and maintained as decided by the anesthesiologist. After induction, the NMB agent was administered and was followed by intubation. Doses of NMB agent were administered intra-operatively as deemed clinically appropriate by the anesthesiologist. The patient was extubated and discharged to the PACU following administration of the NMB reversal agent. Discharge from the PACU occurred when the modified Aldrete score was 9 or 10 and the anesthesiologist approved PACU discharge. ${ }^{3}$ This investigator-initiated study was approved on 13 November 2017 by the Western Institutional Review Board (approval number 20172660).

Eligible patients received rocuronium or vecuronium as NMB and received neostigmine or sugammadex for reversal. Patients were included in the study if they appeared on the electronic medical record list of surgical cases. Data were abstracted from the electronic medical record system (including scanned paper charts). Patients less than 18 years of age and pregnant or breastfeeding women were excluded. Patients with an American Society of Anesthesiologists (ASA) physical status classification score of IV or greater were also excluded. ${ }^{4}$ The inclusion of patients with life-threatening conditions (ASA IV) would have likely increased the variability in OR and/or PACU times due to factors unrelated to the choice of NMB reversal agent.

We collected the following data: demographic information, vitals, ASA scores, time to discharge from the OR, time to discharge from the PACU, bradycardia, and post-operative nausea and/or vomiting (PONV). The dose, agent used, and administration times of the NMB and reversal agent were also collected.

The primary outcome was defined as time from sugammadex or neostigmine administration to OR exit. OR exit was defined as the wheels of the patient's bed exiting the OR in transport to the PACU. Secondary outcomes included overall OR time, overall PACU time, time from reversal agent administration to PACU discharge, as well as the incidence of bradycardia or PONV. Chi-squared test and Fisher's exact test were used as appropriate for nominal data. The Mann-Whitney $U$ test was used for continuous and ordinal data. Continuous data were assumed to be non-normally distributed. Mulitvariable linear regression models were also constructed adjusting for inpatient procedures, age, sex, body mass index, and ASA score. An alpha of 0.05 was utilized for significance testing. All analyses were conducted using Stata 15.1 (College Station, TX).

\section{Results}

The cohort included 277 patients (neostigmine $=143$, sugammadex $=134$ ) after applying the inclusion/exclusion criteria. Reasons for cohort exclusion were the following: ASA score of IV or greater $(n=56)$, spontaneous recovery $(n=38)$, incomplete records ( $n=10)$, pregnancy $(n=3)$, and age $<18$ years $(n=3)$. The types and numbers of surgical procedures are provided in the supplementary tables. Baseline characteristics including age, sex, weight, height, and ASA score were similar for both groups (Table 1 ). The sugammadex group received rocuronium more often for $\mathrm{NMB}(15 \%$ vs. $6 \%, \mathrm{p}=0.02)$. Patients who received neostigmine were administered a median dose of $4 \mathrm{mg}$ (interquartile range [IQR] 3, 5), which is $0.043 \mathrm{mg} / \mathrm{kg}$ (IQR $0.031,0.054)$. All patients who received neostigmine also received glycopyrrolate at a median dose of $0.044 \mathrm{mg} / \mathrm{kg}$ (IQR $0.031,0.058)$. Patients who received sugammadex were administered a median dose of $200 \mathrm{mg}$ (IQR 150, 200), which is $2.06 \mathrm{mg} / \mathrm{kg}$ (IQR 1.87, 2.33).

The median time from NMB reversal agent administration to OR discharge was similar for both neostigmine and sugammadex (16 vs. 15.5 minutes, $p=0.23$ ), Table 2 . Male sex was the only factor significantly associated with time from NMB reversal agent administration to OR discharge in the univariable analysis $(p=0.02)$. Analysis of total OR time (108 vs. 104.5 minutes, $p=$ 0.11 ), total PACU time (113 vs. 113.5 minutes $p=0.58$ ), and time from NMB reversal agent administration to PACU discharge (130 vs. 129.5 minutes, $p=0.33$ ) were similar for both groups.

Time from sugammadex administration to OR discharge was significantly shorter in the linear regression model (mean difference -2.7 minutes, $95 \% \mathrm{Cl}-5.2$ to -0.2 minutes), Table 3 . The model included adjustments for inpatient procedures, age, male sex, body mass index, and ASA score. No other factors included in the model were associated with time from NMB reversal agent administration to OR discharge. Total PACU time was shorter with sugammadex in the multivariable model, but the estimate was imprecise given the wide confidence interval (mean difference -10.0 minutes, $95 \% \mathrm{Cl}-26.3$ to 6.4 minutes). Inpatient surgical procedures were associated with total PACU time in the model (mean difference 25.5 minutes, $95 \% \mathrm{Cl} 8.6$ to 42.5 minutes).

The frequency side effects collected in the cohort were statistically similar between both groups. Sugammadex had numerically lower incidence of bradycardia (5.6\% vs. 2.2\%, OR $0.39,95 \% \mathrm{Cl} 0.11$ to 1.38$)$ and PONV (17.5\% vs. $11.3 \%$, OR 0.60 , $95 \% \mathrm{Cl} 0.30$ to 1.19$)$. No patients in either group experienced refractory NMB.

\section{Discussion}

This study was designed to determine whether sugammadex produced shorter times from NMB reversal agent administration to OR discharge than neostigmine. Sugammadex reduced adjusted OR discharge times after reversal agent administration by three minutes. Sugammadex did not provide 
a statistically significant decrease in total PACU time, PONV, or bradycardia even though each of these outcomes numerically favored sugammadex.

The results suggesting shorter OR exit times with sugammadex were unable to be replicated in the community hospital studied. This may be due to the wide variety of surgeries represented in the study cohort. We included both elective and non-elective procedures unlike randomized controlled trials. The study cohort also had several orthopedic and women's health related surgeries that are not included in studies analyzed by previous meta-analyses. A recent meta-analysis of two randomized controlled trials $(n=282)$ also found sugammadex shortened OR discharge times by six minutes. ${ }^{2}$ One of the studies only evaluated elective procedures and the other only evaluated appendectomies or laparoscopic cholecystectomies.

A retrospective audit was conducted to evaluate the impact of restricting sugammadex on clinical outcomes. Sugammadex had been used in the institute unrestrictedly due to superior reversal and subsidized cost, but upon removal of cost subsidies, guidelines were developed for the state wide use of sugammadex in a restricted manner. This study, $(n=2649)$ of unrestricted and restricted sugammadex also found no difference in total OR time (135 vs. 137 minutes, $p=0.14$ ) similar to the findings of the present study. ${ }^{5}$ This study evaluated patients who underwent a wide variety of surgical procedures, similar to this cohort. The study did not evaluate the impact of sugammadex restriction on time from NMB reversal agent to OR discharge. The meta-analysis by Carron et al found that sugammadex shortened total OR time by a mean difference of 22 minutes. This analysis included fewer patients $(n=108)$ than the current cohort. ${ }^{2}$

The results of the present study agree with others suggesting that sugammadex has shorter total PACU times, but the multivariable model was too imprecise to demonstrate statistical significance. This is in part due to the small sample size of the study cohort and a lower mean difference in total PACU time than previous studies. All four individual studies in the Carron et al meta-analysis $(n=353)$ found shorter PACU times with sugammadex. ${ }^{2}$ The magnitude and precision of this finding was highly dependent on the model used. A fixed effect model (mean difference 32 minutes, $95 \% \mathrm{Cl} 30.6$ to 33.0 minutes) showed a greater impact of sugammadex than a random effects model (mean difference 17 minutes, $95 \% \mathrm{Cl} 0.23$ to 33.7 minutes). Similarly, Rao Kadam and Howell found that unrestricted sugammadex use shortened PACU time by 19 minutes $(p<0.0001) .{ }^{5}$ This study's findings were most similar to total PACU times observed in the present study. This is likely due to the inclusion of multiple surgical types. Carron and colleagues found similar times from NMB reversal agent administration to PACU discharge for both agents. ${ }^{2}$
The rates of PONV and bradycardia were numerically lower for sugammadex in this study's cohort. The differences did not reach the level of statistical significance. Hristovska and colleagues found a significantly lower incidence of bradycardia $(n=1218 ;$ RR $0.16,95 \% C l 0.07$ to 0.34$)$ and PONV ( $n=389 ; R R$ $0.52,95 \% \mathrm{Cl} 0.28$ to 0.97$)$. The authors did not report absolute incidence for these adverse events. ${ }^{1}$ Rao Kadam and Howell observed similar rates of nausea for both agents $(p=0.57)$. The authors did not evaluate vomiting or bradycardia. ${ }^{5}$ The rate of nausea $(2.2 \%)$ they observed was much lower than this study's cohort.

The routine clinical use of sugammadex for NMB reversal in all surgical patients is not justified by the findings from this cohort. The cost of sugammadex for 100 patients is approximately $\$ 9,200$ (US Dollar) at the study institution. The three minutes of OR time gained by using sugammadex is unlikely to be sufficient to allow any extra surgical cases per day. At the study institution, operating room time cost to the facility per minute ranges from \$9-15 per minute depending on the level of acuity. For 100 patients, saving 270 minutes of OR time would result in savings of $\$ 2,430-4,050$. The small absolute differences in rates of PONV and bradycardia are also unlikely to result in sufficient cost savings to justify routine sugammadex use in a broad population like the current cohort. Specifically, the 6.2\% absolute difference in PONV found in this study would result in an estimated increased cost of $\$ 465$ (US Dollar) associated with neostigmine per 100 patients ( $\$ 75$ per patient times $6.2 \%$ increased risk of PONV). ${ }^{6}$

A significant limitation of the present analysis is the lack of Train-of Four monitoring. This made it impossible to judge the depth of NMB produced or whether or not patients achieved complete reversal upon OR exit or PACU discharge. There is a growing emphasis on having mandatory Train-of Four monitoring for all patients undergoing surgical procedures involving NMB. We utilized patients who underwent procedures in 2017, prior to the publication of the consensus document from the International Anesthesia Research Society and the Canadian Anesthesiologists' Society guidelines recommending quantitative monitoring in all patients receiving NMBs. ${ }^{7,8}$ The American standards remain silent on this issue to date. ${ }^{9}$

The generalizability of this study is also limited by being a small, single center, retrospective evaluation that is subject to measured and unmeasured confounding. This study may have more external validity than randomized clinical trials of sugammadex. This is because the study cohort included a wide variety of procedures and few exclusion criteria. Discharge planning or transfer plans may have influenced PACU times. The median neostigmine doses in the study cohort were lower than all but one trial cited by the Carron et al meta-analysis. This may have accentuated the effectiveness of sugammadex while minimizing its safety advantages. The lower neostigmine doses may also represent few patients receiving deep NMB. Reversal 
of deep NMB may be an area where sugammadex has a greater impact on outcomes. ${ }^{2}$ The study's small sample size also affected the precision of the estimates regarding sugammadex's impact on adverse events. A larger study may have found a statistically significant difference. More patients received vecuronium with sugammadex in this cohort $(n=114)$ than all previous trials combined in a 2017 meta-analysis ( $\mathrm{n}=$ 74). ${ }^{10}$ We did not find any impact of vecuronium on the time to OR or PACU discharge. This is not surprising based on a similar duration of action reported for rocuronium $(0.6-0.9 \mathrm{mg} / \mathrm{kg})$ and vecuronium $(0.1 \mathrm{mg} / \mathrm{kg})$ in a randomized study of rapidsequence induction of anesthia. ${ }^{11}$.

NMB reversal agent administration to OR discharge times were better with sugammadex than neostigmine for surgical patients. This difference was not clinically meaningful in the study cohort and was shorter than previously reported by other meta-analyses. This conclusion is limited by the dose of neostigmine being lower than the dose used in other studies, other factors that could affect OR or PACU discharge, and the potential variability of charting adverse events. We recommend using neostigmine for most surgical patients despite the limitations of this trial since sugammadex did not produce a meaningful improvement in the study cohort. Confirmatory studies are needed.

Acknowledgements: The authors would like to acknowledge the contributions of Anusha Ponnuru, Tejal Rana, Tamis Walker, and Hannah Wolf for editorial assistance with this work.

Funding Statement: Support provided solely from institutional/departmental sources

Conflicts of interest: RGH: Grant funding (Merck, Medical Titan Group), All others: No competing interests.

Treatment of Human Subjects: This investigator-initiated study was approved on 13 November 2017 by the Western Institutional Review Board (approval number 20172660).

\section{References}

1. Hristovska AM, Duch $P$, Allingstrup $M$, Afshari A. The comparative efficacy and safety of sugammadex and neostigmine in reversing neuromuscular blockade in adults. A Cochrane systematic review with meta-analysis and trial sequential analysis. Anaesthesia.

2018;73(5):631-641.

2. Carron M, Zarantonello F, Lazzarotto N, Tellaroli P, Ori C. Role of sugammadex in accelerating postoperative discharge: A meta-analysis. J Clin Anesth. 2017;39:38-44.

3. Aldrete JA. The post-anesthesia recovery score revisited. J Clin Anesth. 1995;7(1):89-91.

4. American Society of Anesthesiologists' House of Delegates/Executive Committee. ASA physical status classification system. Available at: https://www.asahq.org/standards-and-guidelines/asaphysical-status-classification-system Accessed: May 1, 2018.

5. Rao Kadam V, Howell S. Unrestricted and Restricted Access to Sugammadex and Side Effect Profile in a Teaching Hospital Centre for Year 2014- Database Audit Study. Anesth Pain Med. 2018;8(1):e63066.

6. Parra-Sanchez I, Abdallah R, You J, et al. A time-motion economic analysis of postoperative nausea and vomiting in ambulatory surgery. Can J Anaesth. 2012;59(4):366375.

7. Dobson G, Chow L, Flexman A, et al. Guidelines to the Practice of Anesthesia - Revised Edition 2019. Can J Anaesth. 2019;66(1):75-108.

8. Naguib M, Brull SJ, Kopman AF, et al. Consensus Statement on Perioperative Use of Neuromuscular Monitoring. Anesth Analg. 2018;127(1):71-80.

9. American Society of Anesthesiologists' Standards and Practice Parameters Committee. Standards for Basic Anesthetic Monitoring. Available at: https://www.asahq.org/standards-andguidelines/standards-for-basic-anesthetic-monitoring Accessed May 1, 2019.

10. Herring WJ, Woo T, Assaid CA, et al. Sugammadex efficacy for reversal of rocuronium- and vecuroniuminduced neuromuscular blockade: A pooled analysis of 26 studies. J Clin Anesth. 2017;41:84-91.

11. Magorian T, Flannery KB, Miller RD. Comparison of rocuronium, succinylcholine, and vecuronium for rapidsequence induction of anesthesia in adult patients. Anesthesiology. 1993;79(5):913-918. 
Table 1: Baseline characteristics

\begin{tabular}{|l|l|l|l|}
\hline Characteristic & $\begin{array}{l}\text { Neostigmine } \\
(\mathbf{n}=\mathbf{1 4 3})\end{array}$ & $\begin{array}{l}\text { Sugammadex } \\
(\mathbf{n}=\mathbf{1 3 4})\end{array}$ & $\mathbf{p}$-value \\
\hline Age & $56(39,69)$ & $58(37,72)$ & 0.56 \\
\hline Male (\%) & 39.2 & 48.5 & 0.12 \\
\hline Height (meters) & $1.7(1.6,1.78)$ & $1.69(1.63,1.78)$ & 0.42 \\
\hline Weight (kilograms) & $88(72,101)$ & $84.5(72,98)$ & 0.25 \\
\hline BMI (kg/m $\left.{ }^{2}\right)$ & $30.1(25.7,35.9)$ & $28.4(25,33.5)$ & 0.06 \\
\hline Neuromuscular blocker used (\%) & & & 0.02 \\
\hline Rocuronium & 6.3 & 14.9 & \\
\hline Vecuronium & 93.4 & 85.1 & \\
\hline Inpatient surgery (\%) & 51.8 & 53.0 & 0.84 \\
\hline ASA score (\%) & & & 0.31 \\
\hline I & 2.8 & 6.0 & \\
\hline II & 54.6 & 57.5 & \\
\hline III & 42.7 & 36.6 & \\
\hline
\end{tabular}

Data presented as median (interquartile range) unless otherwise noted.

Table 2: Univariable analysis of time from drug administration to leaving the operating room

\begin{tabular}{|l|l|l|l|}
\hline Characteristic & No & Yes & p-value \\
\hline Sugammadex & $16(11,23)$ & $15.5(11,20)$ & 0.23 \\
\hline Age 60 years or greater & $16(12,21)$ & $16(10.5,21)$ & 0.77 \\
\hline Male & $15(10,20)$ & $17(14,22)$ & 0.02 \\
\hline Height 1.7 meters or greater & $15(10,20)$ & $17(13,22)$ & 0.09 \\
\hline Weight 80 kilograms or greater & $16(10,20)$ & $16(12,21)$ & 0.14 \\
\hline BMI 30 kg/m or greater & $17(11,20)$ & $15.5(12,21)$ & 0.77 \\
\hline Rocuronium & $16(11,20.5)$ & $16(11,22)$ & 0.65 \\
\hline Inpatient surgery & $17(11,21)$ & $15.5(11.5,20)$ & 0.51 \\
\hline ASA score & & & 0.78 \\
\hline I & & $17.5(14,20)$ & \\
\hline II & & $16(12,21)$ & \\
\hline III & & $16(10,21)$ & \\
\hline
\end{tabular}

Data presented as median (interquartile range) unless otherwise noted.

Table 3. Multivariable linear regression analyses evaluating the impact of sugammadex on time in the operating room or post-anesthesia care unit

\begin{tabular}{|l|l|l|}
\hline & $\begin{array}{l}\text { Time from drug } \\
\text { administration to leave } \\
\text { operating room (minutes) }\end{array}$ & $\begin{array}{l}\text { Total time in post-anesthesia } \\
\text { care unit (minutes) }\end{array}$ \\
\hline Characteristic & $\begin{array}{l}\text { Coefficient }(95 \% \text { Confidence } \\
\text { Interval) }\end{array}$ & $\begin{array}{l}\text { Coefficient (95\% Confidence } \\
\text { Interval) }\end{array}$ \\
\hline Inpatient procedure & $-1.7(-4.4$ to 0.9$)$ & $25.5(8.6$ to 42.5$)$ \\
\hline $\begin{array}{l}\text { Age (per one year } \\
\text { increment) }\end{array}$ & $0.0(-0.1$ to 0.1$)$ & $0.1(-0.5$ to 0.6$)$ \\
\hline Male sex & $2.2(-0.3$ to 4.8$)$ & $-3.4(-19.9$ to 13.1$)$ \\
\hline $\begin{array}{l}\text { Body mass index (per } 1 \\
\text { kg/m }{ }^{2} \text { increment) }\end{array}$ & $0.4(-0.1$ to 0.2$)$ & $0.8(-0.2$ to 1.8$)$ \\
\hline ASA score & $-1.1(-3.7$ to 1.5$)$ & $10.7(-6.1$ to 27.4$)$ \\
\hline Sugammadex & $-2.7(-5.2$ to -0.2$)$ & $-10.0(-26.3$ to 6.4$)$ \\
\hline
\end{tabular}

